\title{
University of Malaya Dental Students' Perception on Competency Based Test
}

\author{
Arbi NA ${ }^{1}$, Mohd Zulkiflee $\mathrm{AH}^{1}$, Gonzalez $\mathrm{MA}^{2 *}$ \\ ${ }^{1}$ Faculty of Dentistry, University of Malaya, Kuala Lumpur, Malaysia \\ ${ }^{2}$ Department of Restorative Dentistry Faculty of Dentistry, University of Malaya, Kuala Lumpur, Malaysia
}

\begin{abstract}
This study was undertaken to explore the perception of Year IV and Year V University of Malaya dental students on different domains related to the competency based test (CBT). Methodology: Aquestionnaire was constructed and a survey conducted to explore the students' perception on four domains related to the competency test: 1 ) benefits, 2) readiness to take, 3) timing and 4) lecturers' supervision. Results: In general, majority of the students agreed that they benefited from the competency test and felt adequately prepared for each test. Students' agreeing on feeling confident to sit for each test ranged from $70.0 \%$ 81.4\% for Year IV and 45.1\%-70.4\% for Year V. Majority of Year IV and Year V students perceived that the timing of each competency test was 'just right'. There was a high response of 'Strongly agree/agree' to availability of patients for practice and assessment, self-confidence and amount and distribution of clinical session as factors affecting when they take the CBT. Majority of Year IV students agreed that the assessment of lecturers was consistent for each tests while Year $V$ had a high percentage of 'Neutral' response. Students' agreeing that there was feedback after the test was $52.9 \%-71.4 \%$ for Year IV and $31.0 \%-56.5 \%$ for Year V. 'Strongly agree/agree' response of $68.6 \%-75.0 \%$ for Year IV and $36.6 \%-52.1 \%$ for Year $V$ was recorded for the level of supervision being the same after passing the test. Conclusion: Overall, majority of students gave positive perception towards CBT in relation to four main domains explored in this research.
\end{abstract}

Keywords: Competency based test, dental clinicians, dental curriculum, dental education, students' perception, University of Malaya

\section{INTRODUCTION}

A pioneer in training dentist to fulfill the need of the country, the Faculty of Dentistry of the University of Malaya was established in 1971.The first batch of intake was in 1972 (1). Since its establishment, the undergraduate curriculum has undergone several changes. The traditional approach to dental education was mainly discipline based. However, starting with students from batch 2010/2015, the Faculty of Dentistry University of Malaya changed to an integrated curriculum and incorporated competency based driven learning and assessment. Students are expected to pass competency tests which measure their level of competence on different cognitive, psychomotor and affective domains $(2,3)$. It is now a global trend among dental schools to change their curriculum from discipline based to competency 
based $(2,3)$. Discipline based curriculum, however, is still dominant especially in developing countries $(2,3)$.

What is competency? In general, it is the capacity to identify a problem and act skilfully in its solution (4). It means being able to function, display psychomotor skills, have knowledge of when and how to use it, demonstrate the values of the profession and communicate knowledge and values in a specific situation (5). It is the behaviour expected of the safe new practitioner (5). It is important because they ensure that dental graduates have a defined level of competence upon graduation. What differentiates competency based learning from traditional approach is that it is believed to be a more 'natural' approach, in which skills are learned as one unit where it does not separate theory from practise (6). Additionally, if the student does not master a skill, he is not allowed to move on to more complicated case. Hence, the timing of student progression is more natural. In traditional discipline-based learning program, it is a time-tabling based curriculum where it works around convenience and efficiency instead of mastery (6). Similarities between both learning program are: use of a variety of teaching methods and the ability to address a variety of learning skills (6).

With changes in the curriculum, it is important to evaluate and get students' perspective as they are the target consumer' (7). Therefore this study was performed with the purpose of exploring dental students' perception of competency tests taken from Year III to Year IV. The four domains explored were: 1) benefits of taking competency test, 2) readiness to take competency test, 3) timing of taking competency test and 4) lecturers' supervision after passing a competency test. This research gives the faculty the opportunity to obtain information on students' perception that serve as feedback to the recently implemented competency based curriculum.

\section{MATERIALS AND METHODS}

A self-administered questionnaire was formulated to explore dental students' perception on four main domains: 1) benefits of taking competency test, 2) readiness to take competency test, 3 ) timing of taking competency test and 4) expectations of lecturers' supervision after passing a competency test. Year IV and Year $V$ dental students from academic year 2014/2015 were invited to participate in the research. Participants' age, gender and ethnicity were the demographic data. Likert scales were utilised with scale of 1 to 6 (1-strongly agree, 2-agree, 3-neutral, 4-disagree, 5-strongly disagree, 6- non applicable) for domains of benefits of competency test and readiness to take competency test. Scales with options 1-very early, 2-early, 3-just right, 4-late, 5-very late and 6-non applicable were used for the timing of competency test. On expectations of lecturer's supervision after the competency test, an answer box was given for students to write their answer. Comment boxes were provided in every section for students to write their comments and opinions. Particulars concerning the questionnaire were discussed with the research supervisor and a pilot test was performed involving two students from Year IV and Year V. Ethical approval was granted by Medical Ethics Committee, Faculty of Dentistry, University of Malaya (reference number: DF RD1404/0023(U)).

The questionnaire was distributed during the fifth week of Sem. 1.1, academic year 2014/2015 and collected in the same week. Participation was voluntary and informed consent from participants was taken. The data was entered and descriptive analysis was performed using SPSS version 12.0.1. The data was tabulated by frequency and percentage. For sections utilising the scale 1-strongly agree, 2-agree, 3-neutral, 4-disagree, 5-strongly disagree and 6-non applicable, scale 1 and 2 were categorised as agreement, 3 as neutral and 4 and 5 as disagreement. For section utilising the scale 1-very early, 2-early, 3-just right, 4-late and 5-very late, scale 1 and 2 were categorised as early, scale 3 as just right scale 4 and 5 as late. Scale 6 was excluded from the data analysis resulting in different frequencies. The answer on expectations of lecturer's supervision after passing the competency test were categorised into yes, no and same in the result tables.

\section{RESULTS}

70 Year IV students and 71 Year V students responded to the questionnaire. There were 53 female students and 17 male students in Year IV while there were 52 female students and 19 male students in Year V. The mean age of Year IV students was 22 while that for Year $\vee$ was 23.

Students' self-perceived benefits of competency test are presented in Table 1. Result of Year IV students show that majority $(57.1 \%$ to $77.9 \%)$ believed each reported benefit in taking competency tests. Majority of Year $\mathrm{V}$ students perceived that they study more than usual due to competency test (74.6\%), allowed to know about own strength and 
weakness $(65.7 \%)$ and had increased discipline in time management (57.1\%). However, less than half perceived that competency test brings about learning at their own pace $(45.7 \%)$, increase in confidence in treating patients after passing the competency test $(47.1 \%)$, that they are able to progress sequentially to more complicated treatment(34.2\%)

Table 1: Year IV and Year V (2014/2015) students' response on benefits of competency test.

\begin{tabular}{|c|c|c|c|c|}
\hline \multirow[t]{3}{*}{ Benefits } & \multicolumn{4}{|c|}{ Response, N (\%) } \\
\hline & $\begin{array}{c}\text { Strongly agree/ } \\
\text { agree }\end{array}$ & \multicolumn{2}{|c|}{ Neutral } & $\begin{array}{l}\text { Strongly } \\
\text { disagree/ } \\
\text { Disagree }\end{array}$ \\
\hline & Year IV Year V & Year IV & Year V & Year IV Year V \\
\hline $\begin{array}{l}1 \text { I study } \\
\text { more than } \\
\text { usual due to } \\
\text { competency } \\
\text { tests. }\end{array}$ & $\begin{array}{cc}53 & 53 \\
(76.8 \%) & (74.6 \%)\end{array}$ & $\begin{array}{c}9 \\
(13.0 \%)\end{array}$ & $\begin{array}{c}14 \\
(19.8 \%)\end{array}$ & $\begin{array}{c}7 \\
(10.1 \%)\end{array}$ \\
\hline $\begin{array}{l}2 \text { I learn at } \\
\text { my own } \\
\text { pace due to } \\
\text { competency } \\
\text { tests. }\end{array}$ & $\begin{array}{cc}44 & 32 \\
(65.7 \%) & (45.7 \%)\end{array}$ & $\begin{array}{c}15 \\
(22.4 \%)\end{array}$ & $\begin{array}{c}26 \\
(37.1 \%)\end{array}$ & $\begin{array}{cc}8 & 12 \\
(11.9 \%) & (17.1 \%)\end{array}$ \\
\hline $\begin{array}{l}3 \text { I am more } \\
\text { discipline } \\
\text { in time } \\
\text { management } \\
\text { due to } \\
\text { competency } \\
\text { tests. }\end{array}$ & $\begin{array}{cc}43 & 40 \\
(64.2 \%) & (57.1 \%)\end{array}$ & $\begin{array}{c}17 \\
(25.4 \%)\end{array}$ & $\begin{array}{c}17 \\
(24.2 \%)\end{array}$ & $\begin{array}{cc}7 & 13 \\
(10.4 \%) & (18.5 \%)\end{array}$ \\
\hline $\begin{array}{l}4 \text { I feel more } \\
\text { confident } \\
\text { in treating } \\
\text { patients } \\
\text { after passing } \\
\text { competency } \\
\text { tests. }\end{array}$ & $\begin{array}{cc}44 & 33 \\
(62.9 \%) & (47.1 \%)\end{array}$ & $\begin{array}{c}19 \\
(27.1 \%)\end{array}$ & $\begin{array}{c}23 \\
(32.8 \%)\end{array}$ & $\begin{array}{cc}7 & 14 \\
(10.0 \%) & (20.0 \%)\end{array}$ \\
\hline $\begin{array}{l}5 \text { I get to know } \\
\text { strength and } \\
\text { weakness } \\
\text { on the } \\
\text { concerned } \\
\text { subject. }\end{array}$ & $\begin{array}{cc}54 & 46 \\
(77.9 \%) & (65.7 \%)\end{array}$ & $\begin{array}{c}8 \\
(11.4 \%)\end{array}$ & $\begin{array}{c}18 \\
(25.7 \%)\end{array}$ & $\begin{array}{c}8 \\
(11.4 \%)\end{array} 6(8.5 \%)$ \\
\hline $\begin{array}{l}6 \text { I can } \\
\text { progress } \\
\text { sequentially } \\
\text { from basic } \\
\text { to more } \\
\text { complicated } \\
\text { treatments } \\
\text { due to } \\
\text { competency } \\
\text { tests. }\end{array}$ & $\begin{array}{cc}40 & 24 \\
(57.1 \%) & (34.2 \%)\end{array}$ & $\begin{array}{c}22 \\
(31.4 \%)\end{array}$ & $\begin{array}{c}26 \\
(37.1 \%)\end{array}$ & $\begin{array}{cc}8 & 20 \\
(11.4 \%) & (28.6 \%)\end{array}$ \\
\hline $\begin{array}{l}7 \text { I feel more } \\
\text { confident } \\
\text { to proceed } \\
\text { to more } \\
\text { complicated } \\
\text { treatments } \\
\text { after passing } \\
\text { competency } \\
\text { tests. }\end{array}$ & $\begin{array}{cc}42 & 30 \\
(60.0 \%) & (42.9 \%)\end{array}$ & $\begin{array}{c}18 \\
(24.7 \%)\end{array}$ & $\begin{array}{c}23 \\
(32.9 \%)\end{array}$ & $\begin{array}{cc}10 & 17 \\
(14.1 \%) & (24.2 \%)\end{array}$ \\
\hline
\end{tabular}

and have increased confidence to proceed to more complicated treatment after passing the competency test $(42.9 \%)$. Year V students responses to 'Neutral' on the benefits of progressing sequentially from basic to more complicated treatments along with benefits of increase in confidence in treating patients and proceeding to more complicated cases ranged from $32.7 \%$ to $37.1 \%$.

Table 2 shows that majority of Year IV students agree that they felt adequately prepared to take the competency tests. 39 out of 42 students $(92.3 \%)$ who took Class III Restoration competency test agreed that they felt adequately prepared and had the highest percentage of agree responses among the competency tests. Oral Health Instruction $(\mathrm{OHI})$ competency test gained the second highest

Table 2: Year IV and Year V (2014/2015) students' response on the statement "I feel adequately prepared for competency tests".

\begin{tabular}{|c|c|c|c|c|c|}
\hline \multirow[t]{2}{*}{$\begin{array}{l}\text { Competency } \\
\text { tests }\end{array}$} & \multicolumn{5}{|c|}{ Response, N (\%) } \\
\hline & $\begin{array}{c}\text { Strongly agree/ } \\
\text { Agree }\end{array}$ & Neutral & & $\begin{array}{l}\text { Stron } \\
\text { disagr } \\
\text { Disag }\end{array}$ & $\begin{array}{l}\text { ngly } \\
\text { gree/ } \\
\text { gree }\end{array}$ \\
\hline & Year IV Year V & Year IV & Year V & Year IV & Year V \\
\hline $\begin{array}{l}1 \text { Scaling \& } \\
\text { Polishing }\end{array}$ & $\begin{array}{cc}50 & 54 \\
(79.3 \%) & (76.1 \%)\end{array}$ & $\begin{array}{c}11 \\
(17.5 \%)\end{array}$ & $\begin{array}{c}14 \\
(19.7 \%)\end{array}$ & $2(3.2 \%)$ & $\begin{array}{c}3 \\
(4.2 \%)\end{array}$ \\
\hline $\begin{array}{l}2 \text { Oral Health } \\
\text { Instruction }\end{array}$ & $\begin{array}{cc}59 & 55 \\
(84.3 \%) & (77.5 \%)\end{array}$ & $\begin{array}{c}8 \\
(11.4 \%)\end{array}$ & $\begin{array}{c}15 \\
(21.1 \%)\end{array}$ & $3(4.3 \%)$ & $\begin{array}{c}1 \\
(1.4 \%)\end{array}$ \\
\hline $\begin{array}{l}3 \text { Local } \\
\text { Anaesthesia } \\
\text { Administration }\end{array}$ & $\begin{array}{cc}55 & 48 \\
(78.6 \%) & (67.6 \%)(\end{array}$ & $\begin{array}{c}12 \\
(17.1 \%)\end{array}$ & $\begin{array}{c}20 \\
(28.2 \%)\end{array}$ & $3(4.3 \%)$ & $\begin{array}{c}3 \\
(4.2 \%)\end{array}$ \\
\hline $\begin{array}{l}4 \text { Class III } \\
\text { Restoration }\end{array}$ & $\begin{array}{cc}39 & 48 \\
(92.3 \%) & (67.6 \%)\end{array}$ & $\begin{array}{c}2 \\
(4.7 \%)\end{array}$ & $\begin{array}{c}18 \\
(25.4 \%)\end{array}$ & $1(2.4 \%)$ & $\begin{array}{c}5 \\
(7.0 \%)\end{array}$ \\
\hline $\begin{array}{l}5 \text { Class II } \\
\text { Slot Cavity } \\
\text { Preparation } \\
\text { (Paediatric } \\
\text { Optech) }\end{array}$ & $\begin{array}{cc}49 & 41 \\
(70.0 \%) & (57.7 \%)\end{array}$ & $\begin{array}{c}16 \\
(22.9 \%)\end{array}$ & $\begin{array}{c}25 \\
(35.2 \%)\end{array}$ & $5(7.2 \%)$ & $\begin{array}{c}5 \\
(7.0 \%)\end{array}$ \\
\hline $\begin{array}{l}6 \text { Endo Optech } \\
\text { on Mounted } \\
\text { Tooth }\end{array}$ & $\begin{array}{cc}49 & 44 \\
(70.0 \%) & (62.0 \%)(\end{array}$ & $\begin{array}{c}14 \\
(20.0 \%)\end{array}$ & $\begin{array}{c}24 \\
(33.8 \%)\end{array}$ & $\begin{array}{c}7 \\
(10.0 \%)\end{array}$ & $\begin{array}{c}3 \\
(4.2 \%)\end{array}$ \\
\hline $\begin{array}{l}7 \text { Primary } \\
\text { Impression }\end{array}$ & $\begin{array}{c}-39 \\
\quad(54.9 \%)\end{array}$ & - & $\begin{array}{c}27 \\
(38.0 \%)\end{array}$ & - & $\begin{array}{c}5 \\
(7.0 \%)\end{array}$ \\
\hline $\begin{array}{l}8 \text { Lateral } \\
\text { Cephalometry }\end{array}$ & $\begin{array}{c}49 \\
-\quad(69.0 \%)\end{array}$ & - & $\begin{array}{c}18 \\
(25.4 \%)\end{array}$ & - & $\begin{array}{c}4 \\
(56.0 \%)\end{array}$ \\
\hline $\begin{array}{l}9 \text { Examination } \\
\text { \& Diagnosis } \\
\text { in Paediatric } \\
\text { Dentistry }\end{array}$ & $\begin{array}{c}42 \\
(59.2 \%)\end{array}$ & - & $\begin{array}{c}22 \\
(31.0 \%)\end{array}$ & - & $\begin{array}{c}7 \\
(9.9 \%)\end{array}$ \\
\hline $\begin{array}{l}10 \text { Extraction of } \\
\text { Single Rooted } \\
\text { Tooth }\end{array}$ & $\begin{array}{c}39 \\
-\quad(65.0 \%)\end{array}$ & - & $\begin{array}{c}19 \\
(31.7 \%)\end{array}$ & - & $\begin{array}{c}2 \\
(3.3 \%)\end{array}$ \\
\hline $\begin{array}{l}11 \text { Extraction of } \\
\text { Multi Rooted } \\
\text { Tooth }\end{array}$ & $\begin{array}{c}34 \\
-\quad(70.8 \%)\end{array}$ & - & $\begin{array}{c}12 \\
(25.0 \%)\end{array}$ & - & $\begin{array}{c}2 \\
(4.2 \%)\end{array}$ \\
\hline
\end{tabular}




\begin{tabular}{|c|c|c|c|c|c|c|}
\hline $\begin{array}{l}12 \text { Class II } \\
\text { Restoration }\end{array}$ & - & $\begin{array}{c}16 \\
(66.7 \%)\end{array}$ & - & $\begin{array}{c}5 \\
(20.8 \%)\end{array}$ & - & $\begin{array}{c}3 \\
(12.5 \%)\end{array}$ \\
\hline $\begin{array}{l}\text { 13 Endo RCT: } \\
\text { up to Canal } \\
\text { Shaping \& } \\
\text { Cleaning }\end{array}$ & - & $\begin{array}{c}43 \\
(60.6 \%)\end{array}$ & - & $\begin{array}{c}21 \\
(29.6 \%)\end{array}$ & - & $\begin{array}{c}7 \\
(9.9 \%)\end{array}$ \\
\hline
\end{tabular}

percentage where 59 out of 70 students (84.3\%) agreed to the statement. The data from Year $\mathrm{V}$ students show that students perceived as adequately prepared to competency tests administered with a range of $57.7 \%$ to $77.5 \%$. Students agreed that they felt adequately prepared in $\mathrm{OHI}(77.5 \%)$ followed by Scaling \& Polishing (S\&P) (76.1\%). 34 out of 46 students who took the Extraction of Multi Rooted Tooth and 16 out of 24 students who took Class II Restoration competency test agreed that they felt prepared for the test. In contrast to Year IV, Year V students gave a high percentage ( $>30 \%$ ) of 'Neutral' response for the following competency tests: Class II Slot Cavity in Paediatric, Primary Impression, Endo Optech on Mounted Tooth, Examination \& Diagnosis (E\&D) in Paediatric Dentistry as well as Extraction of Single Rooted Tooth.

In Table 3, responses of "Strongly agree/ agree" to the statement "I was confident to take the competency tests" for all competency tests administered in Year IV ranged from $70.0 \%$ to $81.4 \% .57$ out of 70 students agreed that they felt confident to take $\mathrm{OHI}$ competency test which ranked in the first place $(81.4 \%)$ followed by S\&P which gets response from 48 out of 64 students who took the test $(75.0 \%) .13$ competency tests are to be passed by Year $V$ students at the end of fourth year. Responses of "Strongly agree/agree" ranged from $45.1 \%$ to $70.4 \%$. Fifty out of $71(70.4 \%)$ students and 49 out of $71(69.0 \%)$ students agreed that they were confident to take the $\mathrm{OHI}$ and S\&P competency tests, respectively. Only 32 out of $71(45.1 \%)$ students agreed that they felt confident to take the Endo Root Canal Treatment (RCT) competency test. Competency tests including Local Anaesthesia Administration, Class III Restoration, Class II Slot Cavity in Paediatric, Endo Optech on Mounted Tooth, Primary Impression, E\&D in Paediatric Dentistry, Class II Restoration and Endo RCT had more than $30 \%$ 'Neutral' responses.

The result from Table 4 shows students' response on timing of each of the competency test administered. Year IV students' responses show that the timing of competency tests administered are 'Just right' ranged from $51.4 \%$ to $64.2 \%$. Meanwhile,
Table 3: Year IV and Year V students' response on the statement "I was confident to take competency tests".

\begin{tabular}{|c|c|c|c|c|c|}
\hline \multirow[t]{3}{*}{$\begin{array}{c}\text { Competency } \\
\text { tests }\end{array}$} & \multicolumn{5}{|c|}{ Response, N (\%) } \\
\hline & $\begin{array}{c}\text { Strongly agree/ } \\
\text { Agree }\end{array}$ & Neutral & & $\begin{array}{l}\text { Stro } \\
\text { disa } \\
\text { Disa }\end{array}$ & $\begin{array}{l}\text { ongly } \\
\text { agree/ } \\
\text { agree }\end{array}$ \\
\hline & Year IV Year V & Year IV & Year V Y & Year IV & / Year V \\
\hline $\begin{array}{l}1 \text { Scaling \& } \\
\text { Polishing }\end{array}$ & $\begin{array}{cc}48 & 49 \\
(75.0 \%) & (69.0 \%)\end{array}$ & $\begin{array}{c}11 \\
(17.2 \%)(\end{array}$ & $\begin{array}{c}19 \\
(26.8 \%)\end{array}$ & $\begin{array}{c}5 \\
(7.8 \%)\end{array}$ & $3(4.2 \%)$ \\
\hline $\begin{array}{l}2 \text { Oral Health } \\
\text { Instruction }\end{array}$ & $\begin{array}{cc}57 & 50 \\
(81.4 \%) & (70.4 \%)\end{array}$ & $\begin{array}{c}9 \\
(12.9 \%)\end{array}$ & $\begin{array}{c}19 \\
(26.8 \%)\end{array}$ & $\begin{array}{c}4 \\
(5.8 \%)\end{array}$ & $2(2.8 \%)$ \\
\hline $\begin{array}{l}3 \text { Local } \\
\text { Anaesthesia } \\
\text { Administration }\end{array}$ & $\begin{array}{cc}51 & 42 \\
(73.9 \%) & (59.2 \%)\end{array}$ & $\begin{array}{c}11 \\
(15.9 \%)\end{array}$ & $\begin{array}{c}25 \\
(35.2 \%)(\end{array}$ & $\begin{array}{c}7 \\
(10.1 \%)\end{array}$ & $4(5.6 \%)$ \\
\hline $\begin{array}{l}4 \text { Class III } \\
\text { Restoration }\end{array}$ & $\begin{array}{cc}31 & 39 \\
(73.8 \%) & (54.9 \%)\end{array}$ & $\begin{array}{c}8 \\
(19.0 \%)\end{array}$ & $\begin{array}{c}26 \\
(36.6 \%)\end{array}$ & $\begin{array}{c}3 \\
(7.1 \%)\end{array}$ & $6(8.5 \%)$ \\
\hline $\begin{array}{l}5 \text { Class II } \\
\text { Slot Cavity } \\
\text { Preparation } \\
\text { (Paediatric } \\
\text { Optech) }\end{array}$ & $\begin{array}{cc}51 & 37 \\
(72.9 \%) & (52.1 \%)\end{array}$ & $\begin{array}{c}12 \\
(17.1 \%)\end{array}$ & $\begin{array}{c}28 \\
(39.4 \%)(\end{array}$ & $\begin{array}{c}7 \\
(10.0 \%)\end{array}$ & $6(8.5 \%)$ \\
\hline $\begin{array}{l}6 \text { Endo Optech } \\
\text { on Mounted } \\
\text { Tooth }\end{array}$ & $\begin{array}{cc}49 & 38 \\
(70.0 \%) & (53.5 \%)\end{array}$ & $\begin{array}{c}18 \\
(25.7 \%)\end{array}$ & $\begin{array}{c}29 \\
(40.8 \%)\end{array}$ & $\begin{array}{c}3 \\
(4.3 \%)\end{array}$ & $4(5.6 \%)$ \\
\hline $\begin{array}{l}7 \text { Primary } \\
\text { Impression }\end{array}$ & $\begin{array}{c}39 \\
(54.9 \%)\end{array}$ & - & $\begin{array}{c}27 \\
(38.0 \%)\end{array}$ & - & $5(7.0 \%)$ \\
\hline $\begin{array}{l}8 \text { Lateral } \\
\text { Cephalometry }\end{array}$ & $\begin{array}{cc}- & 45 \\
& (63.4 \%)\end{array}$ & - & $\begin{array}{c}20 \\
(28.2 \%)\end{array}$ & - & $6(8$ \\
\hline $\begin{array}{l}9 \text { Examination } \\
\text { \& Diagnosis } \\
\text { in Paediatric } \\
\text { Dentistry }\end{array}$ & $\begin{array}{c}37 \\
(52.1 \%)\end{array}$ & - & $\begin{array}{c}28 \\
(39.4 \%)\end{array}$ & - & $6(8.4 \%)$ \\
\hline $\begin{array}{l}10 \text { Extraction of } \\
\text { Single Rooted } \\
\text { Tooth }\end{array}$ & $\begin{array}{c}40 \\
(66.7 \%)\end{array}$ & - & $\begin{array}{c}15 \\
(25.0 \%)\end{array}$ & - & $5(8.3 \%)$ \\
\hline $\begin{array}{l}11 \text { Extraction of } \\
\text { Multi Rooted } \\
\text { Tooth }\end{array}$ & $\begin{array}{c}28 \\
(58.3 \%)\end{array}$ & - & $\begin{array}{c}14 \\
(29.2 \%)\end{array}$ & - & $\begin{array}{c}6 \\
(12.5 \%)\end{array}$ \\
\hline $\begin{array}{l}12 \text { Class II } \\
\text { Restoration }\end{array}$ & $\begin{array}{c}14 \\
(58.3 \%)\end{array}$ & - & $\begin{array}{c}8 \\
(33.3 \%)\end{array}$ & - & $2(8.3 \%)$ \\
\hline $\begin{array}{l}13 \text { Endo RCT: } \\
\text { up to Canal } \\
\text { Shaping \& } \\
\text { Cleaning }\end{array}$ & $\begin{array}{c}32 \\
(45.1 \%)\end{array}$ & - & $\begin{array}{c}30 \\
(42.3 \%)\end{array}$ & - & $\begin{array}{c}9 \\
(12.7 \%)\end{array}$ \\
\hline
\end{tabular}

Table 4: Year IV and Year V (2014/2015) students' response on timing of each competency test.

\begin{tabular}{|c|c|c|c|c|c|}
\hline \multirow[t]{3}{*}{$\begin{array}{l}\text { Competency } \\
\text { tests }\end{array}$} & \multicolumn{5}{|c|}{ Response, N (\%) } \\
\hline & Early/ Very early & $\begin{array}{l}\text { Just } \\
\text { right }\end{array}$ & & Late/ Ve & ery late \\
\hline & Year IV Year V & Year IV & Year V & Year IV & Year V \\
\hline $\begin{array}{l}1 \text { Scaling \& } \\
\text { Polishing }\end{array}$ & $\begin{array}{cc}22 & 15 \\
(32.8 \%) & (21.1 \%)\end{array}$ & $\begin{array}{c}43 \\
(64.2 \%)\end{array}$ & $\begin{array}{c}51 \\
(71.8 \%)\end{array}$ & ${ }^{2}{ }^{2} .0 \%$ & $5(7.0 \%)$ \\
\hline $\begin{array}{l}2 \text { Oral Health } \\
\text { Instruction }\end{array}$ & $\begin{array}{cc}29 & 12 \\
(41.4 \%) & (16.9 \%)\end{array}$ & $\begin{array}{c}37 \\
(52.9 \%)\end{array}$ & $\begin{array}{c}55 \\
(77.5 \%)\end{array}$ & $\begin{array}{c}4 \\
(5.7 \%)\end{array}$ & $4(5.6 \%)$ \\
\hline
\end{tabular}




\begin{tabular}{|c|c|c|c|c|c|c|}
\hline $\begin{array}{l}3 \text { Local } \\
\text { Anaesthesia } \\
\text { Administration }\end{array}$ & $\begin{array}{c}24 \\
(34.3 \%)\end{array}$ & $\begin{array}{c}13 \\
(18.3 \%)(\end{array}$ & $\begin{array}{c}41 \\
(58.6 \%)\end{array}$ & $\begin{array}{c}54 \\
(76.1 \%)\end{array}$ & $\begin{array}{c}5 \\
(7.2 \%)\end{array}$ & $4(5.6 \%)$ \\
\hline $\begin{array}{l}4 \text { Class III } \\
\text { Restoration }\end{array}$ & $\begin{array}{c}13 \\
(23.2 \%)\end{array}$ & $\begin{array}{c}16 \\
(22.5 \%)(\end{array}$ & $\begin{array}{c}33 \\
(58.9 \%)\end{array}$ & $\begin{array}{c}42 \\
(59.2 \%)\end{array}$ & $\begin{array}{c}10 \\
(17.8 \%)\end{array}$ & $\begin{array}{c}13 \\
(18.3 \%)\end{array}$ \\
\hline $\begin{array}{l}5 \text { Class II Slot } \\
\text { Cavity } \\
\text { Preparation } \\
\text { (Paediatric } \\
\text { Optech) }\end{array}$ & $\begin{array}{c}24 \\
(34.3 \%)\end{array}$ & $\begin{array}{c}12 \\
(16.9 \%)(\end{array}$ & $\begin{array}{c}41 \\
(58.6 \%)\end{array}$ & $\begin{array}{c}52 \\
(73.2 \%)\end{array}$ & $\begin{array}{c}5 \\
(7.2 \%)\end{array}$ & $7(9.8 \%)$ \\
\hline $\begin{array}{l}6 \text { Endo Optech } \\
\text { on Mounted } \\
\text { Tooth }\end{array}$ & $\begin{array}{c}28 \\
(40.0 \%)\end{array}$ & $\begin{array}{c}13 \\
(15.5 \%)(\end{array}$ & $\begin{array}{c}36 \\
(51.4 \%)\end{array}$ & $\begin{array}{c}56 \\
(78.9 \%)\end{array}$ & $\begin{array}{c}6 \\
(8.5 \%)\end{array}$ & $2(2.8 \%)$ \\
\hline $\begin{array}{l}7 \text { Primary } \\
\text { Impression }\end{array}$ & - & $\begin{array}{c}12 \\
(16.9 \%)\end{array}$ & - & $\begin{array}{c}50 \\
(70.4 \%)\end{array}$ & - & $\begin{array}{c}9 \\
(12.3 \%)\end{array}$ \\
\hline $\begin{array}{l}8 \text { Lateral } \\
\text { Cephalometry }\end{array}$ & - & $\begin{array}{c}9 \\
(12.7 \%)\end{array}$ & - & $\begin{array}{c}58 \\
(81.7 \%)\end{array}$ & - & $4(5.6 \%)$ \\
\hline $\begin{array}{c}9 \text { Examination } \\
\text { \& Diagnosis } \\
\text { in Paediatric } \\
\text { Dentistry }\end{array}$ & - & $\begin{array}{c}12 \\
(16.9 \%)\end{array}$ & - & $\begin{array}{c}52 \\
(73.2 \%)\end{array}$ & - & $7(9.9 \%)$ \\
\hline $\begin{array}{l}10 \text { Extraction of } \\
\text { Single Rooted } \\
\text { Tooth }\end{array}$ & 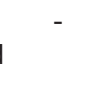 & $\begin{array}{c}8 \\
(13.3 \%)\end{array}$ & - & $\begin{array}{c}41 \\
(68.3 \%)\end{array}$ & - & $\begin{array}{c}11 \\
(18.3 \%)\end{array}$ \\
\hline $\begin{array}{l}11 \text { Extraction of } \\
\text { Multi Rooted } \\
\text { Tooth }\end{array}$ & - & $\begin{array}{c}6 \\
(11.8 \%)\end{array}$ & - & $\begin{array}{c}36 \\
(70.6 \%)\end{array}$ & - & $\begin{array}{c}9 \\
(17.6 \%)\end{array}$ \\
\hline $\begin{array}{l}12 \text { Class II } \\
\text { Restoration }\end{array}$ & - & $\begin{array}{c}2 \\
(6.7 \%)\end{array}$ & - & $\begin{array}{c}16 \\
(53.3 \%)\end{array}$ & - & $\begin{array}{c}12 \\
(40.0 \%)\end{array}$ \\
\hline $\begin{array}{l}\text { 13 Endo RCT: } \\
\text { up to Canal } \\
\text { Shaping \& } \\
\text { Cleaning }\end{array}$ & - & $\begin{array}{c}12 \\
(16.9 \%)\end{array}$ & - & $\begin{array}{c}37 \\
(52.1 \%)\end{array}$ & - & $\begin{array}{c}22 \\
(31.0 \%)\end{array}$ \\
\hline
\end{tabular}

more than a quarter of the students responded that the timing of the tests as 'Early'Nery early' with range from $32.8 \%$ to $41.4 \%$. Year $V$ students' responses showed that the timing of thirteen competency tests administered are 'Just right' ranged from $52.1 \%$ to $81.7 \%$ while responses of 'Very early' and 'Early' ranged from $6.7 \%$ to $22.5 \%$.

Table 5 shows students' response on factors affecting when the competency test is taken. Four factors were listed which include 'patients for practice', 'patients for assessment', 'amount and distribution of clinical sessions' and 'self-confidence'. Among the Year IV students, 'patients for practice' and 'patients for assessment' had response of "Strongly agree/ agree" of more than $80 \%$ while 'self-confidence' and 'amount and distribution of clinical sessions' had a response of $61.4 \%$ and $78.6 \%$ respectively. Year $V$ students gave majority response of 'Strongly agree' and 'Agree' to the following factors; 'patients for practice' (85.9\%), 'patients for assessment' (84.5\%), and 'amount and distribution of clinical sessions' (88.7\%). 'Self-confidence' (49.3\%) had the lowest response.
Table 5: Year IV and Year V (2014/2015) students' response on factors affecting when competency tests are taken.

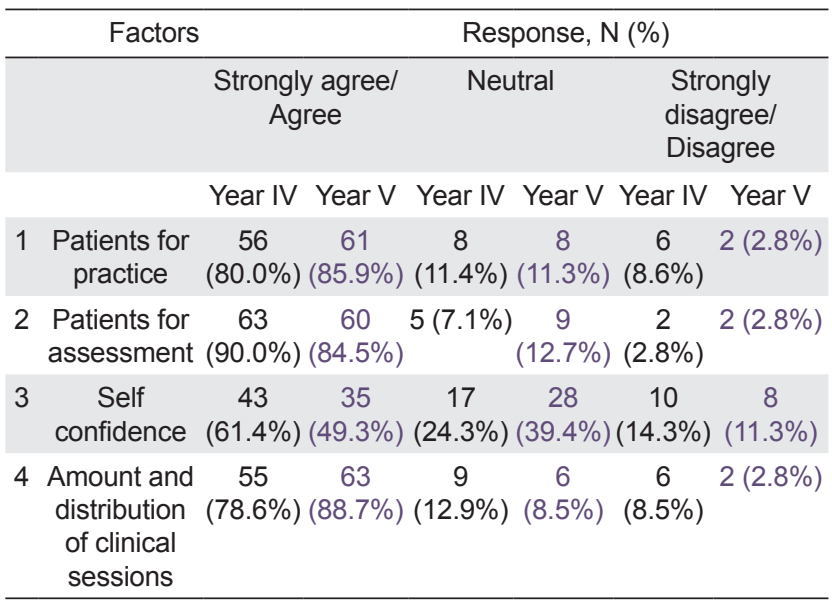

Table 6 shows that majority of the Year IV perceived that assessment by different lecturers during the competency tests were consistent with a range of $64.1 \%$ to $77.1 \%$. The Year $V$ gave a high percentage of "Neutral" response from $40.6 \%$ to $54.2 \%$. Only 4 out of 24 students $(16.7 \%)$ who took Class II Restoration agreed that the assessment was consistent while $10(41.7 \%)$ disagreed with the statement. For Endo RCT, only 14 out of 69 students $(20.3 \%)$ agreed to the statement while $27(39.1 \%)$ disagreed that the assessment was consistent among the lecturers during the test.

Table 6: Year IV and Year V (2014/2015) students' response on the statement "The assessment of lecturers during the competency test is consistent".

\begin{tabular}{|c|c|c|c|c|}
\hline \multirow{3}{*}{\multicolumn{2}{|c|}{$\begin{array}{c}\text { Competency } \\
\text { test }\end{array}$}} & \multicolumn{3}{|c|}{ Response, N (\%) } \\
\hline & & $\begin{array}{l}\text { Strongly agree/ Neutral } \\
\text { Agree }\end{array}$ & Stror & $\begin{array}{l}\text { ongly disagree/ } \\
\text { Disagree }\end{array}$ \\
\hline & & Year IV Year V Year IV & Year V & / Year IV Year V \\
\hline 1 & $\begin{array}{l}\text { Scaling \& } \\
\text { Polishing }\end{array}$ & $\begin{array}{ccc}43 & 24 & 13 \\
(64.1 \%) & (33.8 \%) & (19.4 \%)\end{array}$ & $\begin{array}{c}37 \\
(52.1 \%)\end{array}$ & $\begin{array}{cc}7 & 10 \\
(10.4 \%) & (14.1 \%)\end{array}$ \\
\hline 2 & $\begin{array}{l}\text { Oral Health } \\
\text { Instruction }\end{array}$ & $\begin{array}{ccc}51 & 27 & 11 \\
(72.9 \%) & (38 \%) & (15.7 \%)\end{array}$ & $\begin{array}{c}36 \\
(50,7 \%)\end{array}$ & 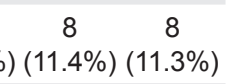 \\
\hline 3 & $\begin{array}{c}\text { Local } \\
\text { Anaesthesia } \\
\text { Administration }\end{array}$ & $\begin{array}{ccc}54 & 27 & 9 \\
(77.1 \%) & (38 \%) & (12.9 \%)\end{array}$ & $\begin{array}{c}38 \\
(53.5 \%)\end{array}$ & $7(10 \%) 6(8,4 \%)$ \\
\hline 4 & $\begin{array}{l}\text { Class III } \\
\text { Restoration }\end{array}$ & $\begin{array}{ccc}28 & 24 & 5 \\
(70.0 \%) & (33.8 \%) & (12.5 \%)\end{array}$ & $\begin{array}{c}30 \\
(42.3 \%)\end{array}$ & $\begin{array}{cc}7 & 17 \\
\text { b) }(17.5 \%) & (23.9 \%)\end{array}$ \\
\hline 5 & $\begin{array}{c}\text { Class II } \\
\text { Slot Cavity } \\
\text { Preparation } \\
\text { (Paediatric } \\
\text { Optech) }\end{array}$ & $\begin{array}{ccc}51 & 26 & 10 \\
(72.9 \%) & (36.6 \%) & (14.3 \%)\end{array}$ & $\begin{array}{c}33 \\
(46.5 \%)\end{array}$ & $\begin{array}{cc}9 & 12 \\
(12.9 \%) & (16.9 \%)\end{array}$ \\
\hline
\end{tabular}




\begin{tabular}{|c|c|c|c|c|c|c|c|}
\hline 6 & $\begin{array}{l}\text { Endo Optech } \\
\text { on Mounted } \\
\text { Tooth }\end{array}$ & $\begin{array}{c}47 \\
(67.1 \%)\end{array}$ & $\begin{array}{c}27 \\
(38.0 \%)(1\end{array}$ & $\begin{array}{c}13 \\
18.6 \%)\end{array}$ & $\begin{array}{c}32 \\
(45 \%)\end{array}$ & $\begin{array}{c}10 \\
(14.3 \%\end{array}$ & $\begin{array}{c}12 \\
(16.9 \%)\end{array}$ \\
\hline 7 & $\begin{array}{l}\text { Primary } \\
\text { Impression }\end{array}$ & - & $\begin{array}{c}19 \\
(26.8 \%)\end{array}$ & - & $\begin{array}{c}35 \\
(49.3 \%)\end{array}$ & - & $\begin{array}{c}17 \\
(23.9 \%)\end{array}$ \\
\hline 8 & $\begin{array}{c}\text { Lateral } \\
\text { Cephalometry }\end{array}$ & - & $\begin{array}{c}32 \\
(45.1 \%)\end{array}$ & - & $\begin{array}{c}33 \\
(46.5 \%)\end{array}$ & - & $6(8.4 \%)$ \\
\hline 9 & $\begin{array}{c}\text { Examination } \\
\& \text { Diagnosis } \\
\text { in Paediatric } \\
\text { Dentistry }\end{array}$ & - & $\begin{array}{c}21 \\
(29.6 \%)\end{array}$ & - & $\begin{array}{c}36 \\
(50,7 \%)\end{array}$ & - & $\begin{array}{c}14 \\
(19.7 \%)\end{array}$ \\
\hline 10 & $\begin{array}{l}\text { Extraction of } \\
\text { Single Rooted } \\
\text { Tooth }\end{array}$ & - & $\begin{array}{c}22 \\
(36.7 \%)\end{array}$ & - & $\begin{array}{c}32 \\
(53.3 \%)\end{array}$ & - & $\begin{array}{c}6 \\
(10.0 \%)\end{array}$ \\
\hline 11 & $\begin{array}{c}\text { Extraction of } \\
\text { Multi Rooted } \\
\text { Tooth }\end{array}$ & - & $\begin{array}{c}16 \\
(33.3 \%)\end{array}$ & - & $\begin{array}{c}26 \\
(54.2 \%)\end{array}$ & - & $\begin{array}{c}6 \\
(12.5 \%)\end{array}$ \\
\hline 12 & $\begin{array}{c}\text { Class II } \\
\text { Restoration }\end{array}$ & - & $\begin{array}{c}4 \\
(16.7 \%)\end{array}$ & - & $\begin{array}{c}10 \\
(41.7 \%)\end{array}$ & - & $\begin{array}{c}10 \\
(41.7 \%)\end{array}$ \\
\hline 13 & $\begin{array}{l}\text { Endo RCT: } \\
\text { up to Canal } \\
\text { Shaping \& } \\
\text { Cleaning }\end{array}$ & - & $\begin{array}{c}14 \\
(20.3 \%)\end{array}$ & - & $\begin{array}{c}28 \\
(40.6 \%)\end{array}$ & - & $\begin{array}{c}27 \\
(39.1 \%)\end{array}$ \\
\hline
\end{tabular}

It is shown in Table 7 that majority $(52.9 \%$ to $71.4 \%$ ) of Year IV students perceived that there is feedback given after the competency tests. For Year $\mathrm{V}$ students, except for Class III Restoration (50.7\%), Class II Restoration (50.0\%) and Endo RCT (56.5\%), the remaining ten competency tests obtained less than $50 \%$ "Strongly agree/agree" response with range from $31.0 \%$ to $45.1 \%$. OHI (31.0\%), Extraction of Single Rooted Tooth (32.3\%) and Primary Impression (33.8\%) had low "Strongly agree/agree" response. The "Neutral" response among Year $\mathrm{V}$ students ranged from $33.3 \%-52.1 \%$.

Table 7: Year IV and Year V (2014/2015) students' response on the statement "There is feedback given after the competency tests".

\begin{tabular}{|c|c|c|}
\hline \multirow{3}{*}{\multicolumn{2}{|c|}{$\begin{array}{l}\text { Competency } \\
\text { tests }\end{array}$}} & Response, N (\%) \\
\hline & & $\begin{array}{l}\text { Strongly agree/ Neutral } \\
\text { Agree }\end{array}$ \\
\hline & & Year IV Year V Year IV Year V Year IV Year V \\
\hline & $\begin{array}{l}\text { Scaling \& } \\
\text { Polishing }\end{array}$ & $\begin{array}{cccccc}50 & 29 & 5 & 31 & 8 & 11 \\
(71.4 \%) & (40.8 \%) & (7.1 \%) & (43.7 \%) & (11.4 \%) & (15.5 \%)\end{array}$ \\
\hline 2 & $\begin{array}{l}\text { Oral Health } \\
\text { Instruction }\end{array}$ & $\begin{array}{cccccc}37 & 22 & 14 & 37 & 19 & 12 \\
(52.9 \%) & (31.0 \%) & (20.0 \%) & (52.1 \%) & (27.2 \%) & (16.9 \%)\end{array}$ \\
\hline 3 & $\begin{array}{l}\text { Local } \\
\text { Anaesthesia } \\
\text { Administration }\end{array}$ & 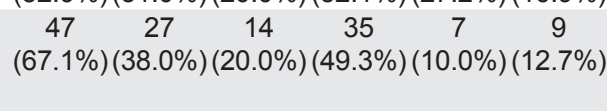 \\
\hline 4 & $\begin{array}{l}\text { Class III } \\
\text { Restoration }\end{array}$ & $\begin{array}{cccccc}28 & 36 & 7 & 26 & 6 & 9 \\
(68.3 \%) & (50.7 \%) & (17.1 \%) & (36.6 \%) & (14.6 \%) & (12.7 \%)\end{array}$ \\
\hline 5 & $\begin{array}{l}\text { Class II } \\
\text { Slot Cavity } \\
\text { Preparation } \\
\text { (Paediatric } \\
\text { Optech) }\end{array}$ & $\begin{array}{cccccc}49 & 25 & 8 & 34 & 11 & 11 \\
(70.0 \%) & (35.7 \%) & (11.4 \%) & (48.5 \%) & (15.8 \%) & (15.7 \%)\end{array}$ \\
\hline
\end{tabular}

\begin{tabular}{|c|c|c|c|c|c|c|c|}
\hline & $\begin{array}{l}\text { Endo Optech } \\
\text { on Mounted } \\
\text { Tooth }\end{array}$ & $\begin{array}{c}48 \\
(68.6 \%\end{array}$ & $\begin{array}{c}29 \\
(40.8 \%)(\end{array}$ & $\begin{array}{c}9 \\
2.9 \%\end{array}$ & $\begin{array}{c}30 \\
(42.3 \%)(\end{array}$ & $\begin{array}{l}13 \\
8.6 \%)\end{array}$ & $\begin{array}{c}12 \\
(16.9 \%)\end{array}$ \\
\hline 7 & $\begin{array}{l}\text { Primary } \\
\text { Impression }\end{array}$ & - & $\begin{array}{c}24 \\
(33.8 \%)\end{array}$ & - & $\begin{array}{c}37 \\
(52.1 \%)\end{array}$ & - & $\begin{array}{c}10 \\
(14,1 \%)\end{array}$ \\
\hline 8 & $\begin{array}{l}\text { Lateral } \\
\text { Cephalometry }\end{array}$ & - & $\begin{array}{c}28 \\
(39.4 \%)\end{array}$ & - & $\begin{array}{c}32 \\
(45.1 \%)\end{array}$ & - & $\begin{array}{c}11 \\
(15.5 \%)\end{array}$ \\
\hline 9 & $\begin{array}{l}\text { Examination } \\
\text { \& Diagnosis } \\
\text { in Paediatric } \\
\text { Dentistry }\end{array}$ & - & $\begin{array}{c}32 \\
(45.1 \%)\end{array}$ & - & $\begin{array}{c}30 \\
(42,3 \%)\end{array}$ & - & $\begin{array}{c}9 \\
\left(12.7^{\circ}\right.\end{array}$ \\
\hline & $\begin{array}{l}0 \text { Extraction of } \\
\text { Single Rooted } \\
\text { Tooth }\end{array}$ & - & $\begin{array}{c}20 \\
(32.3 \%)\end{array}$ & - & $\begin{array}{c}32 \\
(51.6 \%)\end{array}$ & - & $\begin{array}{c}10 \\
(16.1 \%\end{array}$ \\
\hline & $\begin{array}{l}1 \text { Extraction of } \\
\text { Multi Rooted } \\
\text { Tooth }\end{array}$ & - & $\begin{array}{c}20 \\
(42.6 \%)\end{array}$ & - & $\begin{array}{c}22 \\
(46.8 \%)\end{array}$ & - & $\begin{array}{c}5 \\
(10.6 \%\end{array}$ \\
\hline & $\begin{array}{l}2 \text { Class II } \\
\text { Restoration }\end{array}$ & - & $\begin{array}{c}12 \\
(50.0 \%)\end{array}$ & - & $\begin{array}{c}9 \\
(37.5 \%)\end{array}$ & - & $\begin{array}{c}3 \\
(12.5 \%\end{array}$ \\
\hline & $\begin{array}{l}3 \text { Endo RCT: } \\
\text { up to Canal } \\
\text { Shaping \& } \\
\text { Cleaning }\end{array}$ & - & $\begin{array}{c}39 \\
(56.5 \%)\end{array}$ & - & $\begin{array}{c}23 \\
(33.3 \%)\end{array}$ & - & $\begin{array}{c}7 \\
(10.1 \%\end{array}$ \\
\hline
\end{tabular}

In Table 8, Year IV students' response of "Strongly agree/agree" on whether the supervision was the same after passing the competency tests was $68.6 \%$ to $75.0 \%$. Among the Year V students, competency tests including Class III Restoration, Class II Slot Cavity in Paediatric, Primary Impression, Lateral Cephalometry and Class II Restoration had "Strongly agree/agree" responses ranging from $50.7 \%$ to $52.1 \%$. OHI received the lowest "Strongly agree/agree" responses (36.6\%). The competency tests administered among Year $V$ students obtained 'Neutral' response ranging from $36.0 \%$ to $45.1 \%$.

Table 8: Year IV and Year V (2014/2015) students' response on the statement "I think the supervision after taking the competency tests is the same".

\begin{tabular}{|c|c|c|c|c|}
\hline & Competency & Respons & se, $N(\%)$ & \\
\hline & & $\begin{array}{l}\text { Strongly agree/ Neutral } \\
\text { Agree }\end{array}$ & & $\begin{array}{l}\text { Strongly } \\
\text { disagree/ } \\
\text { Disagree }\end{array}$ \\
\hline & & Year IV Year V Year IV & Year V & Year IV Year V \\
\hline 1 & $\begin{array}{l}\text { Scaling \& } \\
\text { Polishing }\end{array}$ & $\begin{array}{ccc}48 & 32 & 8 \\
(68.6 \%) & (45.2 \%) & (11.4 \%)\end{array}$ & $\begin{array}{c}28 \\
(39.4 \%)\end{array}$ & $\begin{array}{cc}7 & 11 \\
(10.0 \%)(15.5 \%)\end{array}$ \\
\hline 2 & $\begin{array}{l}\text { Oral Health } \\
\text { Instruction }\end{array}$ & $\begin{array}{ccc}48 & 26 & 17 \\
(68.6 \%) & (36.6 \%)(24.3 \%)\end{array}$ & $\begin{array}{c}32 \\
(45.1 \%)\end{array}$ & $\begin{array}{cc}5 & 13 \\
(7.2 \%) & (18.3 \%)\end{array}$ \\
\hline 3 & $\begin{array}{l}\text { Local } \\
\text { Anaesthesia } \\
\text { Administration }\end{array}$ & $\begin{array}{ccc}49 & 32 & 11 \\
(70.0 \%) & (45.1 \%)(15.7 \%)\end{array}$ & $\begin{array}{c}29 \\
(40.8 \%)\end{array}$ & $\begin{array}{cc}10 & 10 \\
(14.3 \%)(14.1 \%)\end{array}$ \\
\hline 4 & $\begin{array}{l}\text { Class III } \\
\text { Restoration }\end{array}$ & $\begin{array}{ccc}30 & 36 & 6 \\
(75.0 \%) & (50.7 \%)(15.0 \%)\end{array}$ & $\begin{array}{c}29 \\
(40.8 \%)\end{array}$ & $\begin{array}{cc}4 & 6 \\
(10.0 \%) & (8.4 \%)\end{array}$ \\
\hline 5 & $\begin{array}{l}\text { Class II } \\
\text { Slot Cavity } \\
\text { Preparation } \\
\text { (Paediatric } \\
\text { Optech) }\end{array}$ & $\begin{array}{ccc}51 & 37 & 12 \\
(72.9 \%) & (52.1 \%) & (17.1 \%)\end{array}$ & $\begin{array}{c}28 \\
(39.4 \%)\end{array}$ & $\begin{array}{cc}7 & 6 \\
(10.0 \%) & (8.4 \%)\end{array}$ \\
\hline
\end{tabular}




\begin{tabular}{|c|c|c|c|c|c|c|c|}
\hline 6 & $\begin{array}{l}\text { Endo Optech } \\
\text { on Mounted } \\
\text { Tooth }\end{array}$ & $\begin{array}{c}52 \\
(74.3 \%)\end{array}$ & $\begin{array}{c}34 \\
(47.9 \%)(1\end{array}$ & $\begin{array}{c}13 \\
18.6 \%)\end{array}$ & $\begin{array}{c}30 \\
(42.3 \%)\end{array}$ & $\begin{array}{c}5 \\
(7.1 \%)\end{array}$ & $\begin{array}{c}7 \\
(9.8 \%)\end{array}$ \\
\hline 7 & $\begin{array}{l}\text { Primary } \\
\text { Impression }\end{array}$ & - & $\begin{array}{c}36 \\
(50.7 \%)\end{array}$ & - & $\begin{array}{c}28 \\
(39.4 \%\end{array}$ & - & $\begin{array}{c}7 \\
(9.8 \%)\end{array}$ \\
\hline 8 & $\begin{array}{l}\text { Lateral } \\
\text { Cephalometry }\end{array}$ & - & $\begin{array}{c}36 \\
(50.7 \%)\end{array}$ & - & $\begin{array}{c}27 \\
(38.0 \%)\end{array}$ & - & $\begin{array}{c}8 \\
(11.2 \%)\end{array}$ \\
\hline 9 & $\begin{array}{l}\text { Examination } \\
\text { \& Diagnosis } \\
\text { in Paediatric } \\
\text { Dentistry }\end{array}$ & - & $\begin{array}{c}34 \\
(47.9 \%)\end{array}$ & - & $\begin{array}{c}30 \\
(40.3 \%)\end{array}$ & - & $\begin{array}{c}7 \\
(9.8 \%)\end{array}$ \\
\hline & $\begin{array}{l}\text { Extraction of } \\
\text { Single Rooted } \\
\text { Tooth }\end{array}$ & - & $\begin{array}{c}26 \\
(43.3 \%)\end{array}$ & - & $\begin{array}{c}27 \\
(45.0 \%)\end{array}$ & - & $\begin{array}{c}7 \\
(11.7 \%)\end{array}$ \\
\hline & $\begin{array}{l}\text { Extraction of } \\
\text { Multi Rooted } \\
\text { Tooth }\end{array}$ & - & $\begin{array}{c}24 \\
(49.0 \%)\end{array}$ & - & $\begin{array}{c}19 \\
(38.8 \%)\end{array}$ & - & $\begin{array}{c}6 \\
(12.2 \%)\end{array}$ \\
\hline & $\begin{array}{l}\text { Class II } \\
\text { Restoration }\end{array}$ & - & $\begin{array}{c}13 \\
(52.0 \%)\end{array}$ & - & $\begin{array}{c}9 \\
(36.0 \%)\end{array}$ & - & $\begin{array}{c}3 \\
(12.0 \%)\end{array}$ \\
\hline & $\begin{array}{l}\text { Endo RCT: } \\
\text { up to Canal } \\
\text { Shaping \& } \\
\text { Cleaning }\end{array}$ & - & $\begin{array}{c}31 \\
(45.6 \%)\end{array}$ & - & $\begin{array}{c}27 \\
(39.7 \%)\end{array}$ & - & $\begin{array}{c}10 \\
(14.7 \%)\end{array}$ \\
\hline
\end{tabular}

The response on whether the supervision should be the same after taking the competency test is shown in Table 9. More than half of Year IV and Year $V$ students believed that the amount of supervision should be the same after passing a competency test. The reasons (Table 10) given are; 'because different cases have different level of difficulty', 'as a learning process', 'to be fair to all students', 'to maintain the quality of works', and 'because students tend to make mistake' Meanwhile, $24.3 \%$ of Year IV and $33.8 \%$ of Year $V$ students stated that the amount of supervision should be reduced.

Table 9: Year IV and year V (2014/2015) students' response on amount of supervision that should be given after passing competency tests.

\begin{tabular}{|c|c|c|c|}
\hline & \multirow{2}{*}{$\begin{array}{c}\text { Amount of supervision that } \\
\text { should be given after passing } \\
\text { competency tests }\end{array}$} & \multicolumn{2}{|c|}{ Response, N (\%) } \\
\hline & & Year IV & Year V \\
\hline 1 & $\begin{array}{l}\text { Amount of supervision should } \\
\text { be the same }\end{array}$ & $36(51.4 \%)$ & $36(50.7 \%)$ \\
\hline 2 & $\begin{array}{l}\text { Amount of supervision should } \\
\text { be reduced }\end{array}$ & $17(24.3 \%)$ & $24(33.8 \%)$ \\
\hline 3 & No answer & $17(24.3 \%)$ & $11(15.5 \%)$ \\
\hline
\end{tabular}

Table 10: Students' comments on why they believed level of supervision after passing competency tests should be the same.

\begin{tabular}{lccc}
\hline & Students' comments & \multicolumn{2}{c}{ Response, N (\%) } \\
\cline { 3 - 4 } & Year IV & Year V \\
\hline 1 Because different cases have different $6(8.4 \%)$ & $5(7.0 \%)$ \\
level of difficulty. & & \\
& As a learning process. & $3(4.2 \%)$ & $7(9.8 \%)$ \\
2 & To be fair to all students. & $1(1.4 \%)$ & $2(2.8 \%)$ \\
3 & To maintain the quality of works. & $2(2.8 \%)$ & $6(8.4 \%)$ \\
4 & Because students tend to make & $2(2.8 \%)$ & - \\
\hline 5 & mistake. & & \\
\hline
\end{tabular}

\section{DISCUSSION}

With the implementation of the new curriculum in our faculty, we wanted to know how the students felt about certain aspects of the competency test. We designed a questionnaire with the help of the research supervisor to explore students' perceptions. The questionnaire was constructed and pre-tested on two Year IV and Year V students. No modification was made after the pre-test. We planned to administer the questionnaire at the end of Semester 2 for the academic year 2013/2014 after the final examinations. This was to ensure that all students completed the competency tests by the end of their Year III and Year IV and that it did not interfere with their final examinations (8). However, due to some unforeseen circumstances, it was delayed to the 5th week of the next semester in academic year 2014/2015. Students' perceptions on benefits, timing, preparedness and supervision of competency tests were explored using this questionnaire.

In Oxford English Dictionary, perception is defined as a late Middle English word originating from the Latin word 'perceptio'. It means "the way in which something is regarded, understood or interpreted, intuitive understanding and insight" (9). Students' perception of the learning environment is crucial and their perception on the characteristics of learning environment can affect students' approach to learning as well as the quality of learning outcome (10). Perceptions of dental students concerning the curriculum are important. Students' opinions regarding the new curriculum may provide insight to the faculty on the implementation of the CBT. Though the result of students' opinion from one dental school may not extrapolated to all dental schools, the results do generally illustrate possible strengths and weaknesses of the curriculum that need to be explored (11). 
A study was conducted by Bell and Mitchell to illustrate the opinions and perceptions of students under two different curricula. Students under competency based curriculum perceived learning theory and practice as one unit and being equally important. They also felt more responsible in managing their own learning in comparison to those in the traditional learning program (6). Yip and Smales identified that assessment of students' performance in competency based education are effective for dentist to improve knowledge, professional attitudes and performance skills (2). According to Norton (1987), competency based education gives the benefit of achieving competencies required in the performance of a job, builds confidence when achieving a level of competence, provides a list of competencies achieved and results in an efficient and effective use of training time. Aside from these, more training time is devoted to practical learning than lectures and more time is devoted to evaluate each participant's ability to perform essential job skills (12). A good competency based curriculum describes the knowledge and skills that can be applied in complex situations. The skills described remain valuable as time passes even if the knowledge content has changed. A competency based curriculum should have clear learning objectives and performance criteria as well as effective rubrics which can help students identify their performance level and what they need to do and improve. Additionally, the rubric will help them to understand themselves better as learners as well as allow for personalisation and give opportunity for deeper learning. With competency education, students advance upon mastery of a skill, the learning objective empowers them and assessment is meaningful and provides a positive learning experience. Students receive timely support based on their learning need and learning outcomes emphasize competencies that allow students to gain knowledge, apply the knowledge and at the same time develop important skills and deposition (13). Competency based education should be able to motivate learners to acquire knowledge, enable them to self-assess and work in a team. This should reduce the focus on a teacher dependent learning system and promote self-directed learning (14). Therefore in our questionnaire we asked the students that as a benefit of the CBT whether they learn at their own pace, they are more disciplined at time management, they are able to progress sequentially from basic to more complicated treatment procedures and they are more confident to proceed with more complicated treatment procedures after passing the competency tests. We also asked whether they studied more because of the competency test, they get to know their strengths and weaknesses on subjects tested and they became more confident to treat patients after passing the competency test. In competency based education, a student is not allowed to move on to more complicated treatment if he/she does not sufficiently master a specific skill (6). Hence the result shows that majority of Year IV students perceived the they were benefited from CBT in terms of ability to progress sequentially from basic to complicated treatments but less than half of Year $V$ students perceived this. There were a large proportion of "Neutral" responses to this. It may be that they considered the cases they were treating in Year IV was not much more difficult or complicated than those they did in Year III. Wanigasooriya stated that self-confidence is important and act as main role in enhancing the competencies (15). Student with higher self-efficacy were more likely to perform better in clinic (15). From the result, less than half of Year $\mathrm{V}$ students agree that they had increase in confidence in treating patient after passing a test and felt more confident to proceed to more complicated treatment after passing a competency test while majority of Year IV perceived that they benefited in confidence due to CBT. But it needs to be noted that there were more than a quarter of Year $V$ students that took a neutral stance. The Year $V$ is the first batch under the integrated competency based curriculum. The reason for the high percentage of neutral response is unclear and would need further investigation.

Majority of students felt that they were adequately prepared to sit for the competency tests and the "Strongly agree/agree" responses for feeling adequately prepared are higher as compared to confidence to take the competency tests. What we meant with the term 'adequately prepared' was having as much practice and study as necessary while the term confident meant feeling certain about something (9). According to a study, lack of confidence may be related to anxiety rather than due to lack of competence (16). Problems with confidence can be reduced by increasing the exposure to procedures and patients. However, it may not correlate with performance in tests (17). The results may be influenced by students' anxiety to sit for the test. Students commented that they felt adequately prepared the most in $\mathrm{OHI}$ and Scaling \& Polishing. The reason being that they are able to practice on every patient as it is a required procedure.

Timing is defined as the choice, judgement or control of when something should be done (9). A good timing allows students to prepare themselves and allow them to plan their treatment before they sit for competency tests. Majority of Year IV and V students believe that the timing of each competency tests 
administered is just right. Students expressed their concerns that different patients with different level of difficulty affect their timing to take competency tests. The cases referred to undergraduate students are complex and unsuitable (8). This might cause bias on when to take competency tests as two individuals with equal skills do not produce the same probability of success. Students are advised to select a suitable case before sitting for competency test.

The manner in which the clinical sessions are distributed is crucial in order for students to organize their timing to take the competency tests (8). Students mentioned some example as there is too much Removable Prosthetic sessions in Semester 1.1 and the sessions reduced tremendously in Semester 1.2. The way the sessions were distributed affects students' time management and therefore affected the timing for taking competencytests .

Feedback of the results is one of many forms of assessments of student performance. It can provide information to enable further progress to a student and provide summary to students' performance where it shows the results are final (2). Feedback after a competency test is effective for students to improve knowledge, professional attitudes and skills. Majority of Year IV and Year V students indicated that feedback was given after the competency tests except for $\mathrm{OHI}$, primary impression and extraction. $\mathrm{OHI}$ competency test is administered as an OSCE. There is one supervisor in the OHI OSCE station who performs the evaluation. Students were not given feedback after the test due to the examination setting. Students believe that this is not a critical problem because they can improve $\mathrm{OHI}$ even after passing the competency test since they can always perform $\mathrm{OHI}$ on every patient.

The integration of teaching and work in a curriculum allows students to understand the reason of the tasks, why and how the tasks should be carried out. This allows them to work not only on their skills but on their knowledge as well. Supervisors play an important part in students' daily practice. The supervision during and after passing a competency test acts as an important resource for students to identify their problems as well as encourages critical thinking (8). Result shows that majority of Year IV and Year V students agree that there was consistent assessment given by different lecturers for most of the competency tests administered. But there were high percentages of students who disagree upon the consistency of the assessment in Class II Restoration and Endo RCT competency tests. Students expressed their frustration that the assessment during Endo RCT competency test is rather confusing and not the same between two different lecturers. Example given is that there are some students who failed upon a minor fault while there are some students who still passed upon a critical fault during the test. This raised the question of whether the problem arose because of different opinions and experiences of the lecturers or the evaluation system of the competency. The students may not have been familiar with the evaluation system.

The introduction of the competency test has been beneficial to students. It has also been a means to inform the students of their expected knowledge and skills for their graduation. This also gave the students the opportunity for self-directed learning and determines their preparedness to be assessed. Information gathered will assist the administrators in improving the conduct of the competency tests.

This study had limitations. The questionnaire was conducted not at the end of the academic year 2013-2014 when the experience would have been more recent. The recall of factual events that affects perceptions may have been affected. The quantitative data is obtained from the questionnaire does not give the reasons for the responses. Qualitative research to include focused group discussion or interviews of the students should be conducted to find out the reasons for their responses. It is also recommended that the exploration of the perception of these two batches of dental students be continued until their graduation for information on their perception of the whole process of clinical competency testing in their dental education. This can include other aspects or domains that would contribute to the improvement of the training programme.

\section{CONCLUSION}

Implementation of integrated curriculum incorporated with competency based learning has brought about changes in the way students learn. Students' perceptions on CBT provide an opportunity to serve as feedback to the faculty. Overall, majority of Year IV and Year V students gave positive responses towards CBT in relation to the benefits, preparedness, timing and supervision of competency test. Reasons for 'neutral' responses from students need to be investigated. It would be interesting if perceptions of the next batches and the reasons for these perceptions could be explored for further improvement. 


\section{DECLARATION OF INTEREST}

The authors reflect no conflicts of interest.

\section{REFERENCES}

1. Undergraduates Programme: Programme Overview. Faculty of Dentistry, University of Malaya. Web. Available from : http://dentistry. um.edu.my/mainpage.php?module=Maklumat \&kategori=46\&id=11\&papar=1. [Accessed on 10/19/2014].

2. Yip HK, Smales RJ. Review of competency based education. Br Dent J. 2000; 189(6): 3246.

3. Razak IA, Latifah RR, Jaafar N, Abu Hassan $\mathrm{Ml}$, Ab Murat N. Assessing the competence of University of Malaya dental graduates: employers' and graduates' perceptions. J Dent Educ. 2008; 72(3): 364-9.

4. Beltran-Neira RJ, Beltran-Aguilar ED. Taxonomy for competency based dental curricula. J Dent Educ. 2004; 68(9): 978-84.

5. Chambers DW, Gerrow JD. Manual for developing and formatting competency statements. J Dent Educ. 1994; 58: 361-6.

6. Bell JS, Mitchell R. Competency based versus traditional cohort-based technical education: a comparison of students' perceptions. Journal of Career and Technical Education. 2000; 17(1): 5-22.

7. Henzi D, Davis E, Jasinevicus R, Hendricson W. In the students' own words: what are the strengths and weaknesses of the dental school curriculum? J Dent Educ. 2007; 71(5): 632-45.

8. Rolland S, Hobson R, Hanwell S. Clinical competency exercises: some student perceptions. Eur J Dent Educ. 2007; 11(3): 184-91.

9. Oxford English Dictionary. Web. Available from http://www.oxforddictionaries.com/. [Accessed on 11/05/2014].

10. Entwistle N, Tait H. Approaches to learning, evaluations of teaching, and preferences for contrasting academic environments. Higher Education. 1990; 19(2): 169-94.

11. Shetty VB, Shirahatti RV, Pawar P. Students' Perceptions of their education on graduation from a dental school in India. J Dent Educ. 2012; 76(11): 1520-6.

12. Sullivan RS. The competency-based approach to training. JHPIEGO Strategy Paper. 1995; (1): $1-9$.
13. Sturgis $\mathrm{C}$. The art and science of designing competencies. Competency Works Issue Brief. International Association for K-12 Online Learning. 2012. Available from http:// www.competencyworks.org/wp-content/ uploads/2012/08/CompetencyWorks IssueBrief_DesignCompetencies-Aug-2012. pdf. [Accessed on 03/04/2014].

14. Taleghani M, Solomon ES, Wathen WF. Nongraded clinical evaluation of dental students in a competency-based education program. $\mathrm{J}$ Dent Educ. 2004; 68(6): 644-55.

15. Manakil J, George R. Reviewing Competency in dental education. International J Dent Clinics. 2011: 3(2): 33-9.

16. Stewart J, O'Halloran C, Barton JR, Singleton SJ, Harrigan P, Spencer J. Clarifying the concepts of confidence and competence to produce appropriate self-evaluation Measurement Scales. Med Educ. 2000; 34: 903-9.

17. Morgan PJ, Cleave-Hogg D. Comparison between medical students' experience, confidence and competence. Med Educ. 2002; 36(6): 534-9.

\section{Corresponding author:}

\section{Dr Maria Angela Gonzalez}

Department of Restorative Dentistry,

Faculty of Dentistry,

University of Malaya,

50603 Kuala Lumpur,

Malaysia.

Email:gonzalez@um.edu.my 